\title{
Calidad de servicio y satisfacción de pacientes sometidos a tratamientos estéticos faciales mínimamente invasivos de una clínica privada. Lima-Perú. 2019
}

\author{
Karen Lissette Ramos Miranda* 1, a; Luis Enrique Podesta Gavilano 1, b; Raúl Alberto Ruiz Arias 1, c
}

RESUMEN

Objetivo: Establecer la relación entre calidad de servicio y satisfacción en pacientes sometidos a tratamientos estéticos faciales mínimamente invasivos de una clínica privada, Lima-Perú,2019.

Materiales y métodos: Estudio de tipo observacional, descriptivo y transversal con enfoque cuantitativo y correlacional. Se realizó una encuesta a 123 pacientes con el cuestionario para calidad de servicio en base a percepciones (SERVPERF, por sus siglas en inglés) y el cuestionario de satisfacción del usuario de consultas externas (SUCE), que fueron adaptados a la población de estudio. Se utilizó el programa estadístico SPSS 20 y la prueba de correlación de Spearman.

Resultados: El 23,00\% de los pacientes reportó una calidad de atención alta; el 58,00 \%, media; y el 19,00 \%, una atención de calidad baja. Además, la calidad media predomina también en las siguientes dimensiones de calidad de servicio: fiabilidad (49\%), capacidad de respuesta (48,00\%), seguridad (56,00\%), empatía (52,00\%) y aspectos tangibles (57,00\%). Respecto a la satisfacción, el 95 \% tuvo una satisfacción media después de su atención; y el 5,00 \%, una satisfacción baja. Así mismo, en ambas dimensiones de la variable satisfacción (atención administrativa y atención asistencial) se observa un nivel de satisfacción media. Se demostró la relación entre calidad de servicio y satisfacción. De igual forma, se demostró una relación significativa (sig < 0,05) entre las dimensiones fiabilidad, capacidad de respuesta y elementos tangibles de la variable calidad de servicio con la variable satisfacción. Por el contrario, no se encontró una relación significativa $($ sig $>0,05)$ entre la variable satisfacción y dos de las dimensiones de la variable calidad de servicio (seguridad y empatía).

Conclusiones: Existe relación significativa entre la calidad de servicio y la satisfacción en pacientes sometidos a tratamientos estéticos faciales mínimamente invasivos de una clínica privada de Lima (Perú) en 2019.

Palabras clave: Calidad de la atención de salud; Satisfacción del paciente; Estética; Tratamiento conservador (Fuente: DeCS BIREME).

\section{Quality of service and satisfaction among patients who underwent minimally invasive facial cosmetic treatments in a private clinic. Lima-Peru. 2019}

\section{ABSTRACT}

Objective: To establish the relationship between quality of service and satisfaction among patients who underwent minimally invasive facial cosmetic treatments in a private clinic, Lima-Peru, 2019.

Materials and methods: An observational, descriptive and cross-sectional study with a quantitative and correlational approach. One hundred twenty-three (123) patients were surveyed by means of the service performance (SERVPERF) model and the outpatient service user satisfaction (SUCE) questionnaire, which were adapted to the study population. The IBM SPSS StatisticS version 20 and the Spearman's rank correlation test were used.

Results: Twenty-three percent (23\%) of the patients reported a high quality of service; $58 \%$, a medium quality of service; and $19 \%$, a low quality of service. Additionally, a medium-level quality prevailed in the following quality of service dimensions: reliability (49\%), responsiveness (48\%), security (56\%), empathy (52\%) and tangible aspects (57 \%). Regarding satisfaction, $95 \%$ of the patients reported a medium-level satisfaction and $5 \%$, a low-level satisfaction after receiving the service. Likewise, in both dimensions of the satisfaction variable (administrative service and healthcare service), a medium-level satisfaction was observed. The research demonstrated that there is a relationship between quality of service and satisfaction. It also demonstrated that there is a significant relationship (sig < 0.05$)$ between three dimensions of the quality of service variable (reliability, responsiveness and tangible aspects) and the satisfaction variable. Conversely, no significant relationship (sig > 0.05) was found between two dimensions of the quality of service variable (security and empathy) and the satisfaction variable. Conclusions: There is a significant relationship between quality of service and satisfaction among patients who underwent minimally invasive facial cosmetic treatments in a private clinic, Lima-Peru, 2019.

Keywords: Quality of health care; Patient satisfaction; Esthetics; Conservative treatment (Source: MeSH NLM).

1 Universidad Nacional Mayor de San Marcos. Lima, Perú.

a Bachiller en Obstetricia.

b Doctor en Ciencias de la Salud.

c Magíster en Estadística.

* Autor corresponsal 


\section{INTRODUCCIÓN}

En los últimos años, el mundo se ha desarrollado a gran velocidad, pues tanto las oportunidades como los retos en tecnología, administración y calidad han aumentado con el paso de los años. Debido a ello, las organizaciones se enfrentan a la necesidad de perfeccionar la calidad, ya sea en su producto o servicio. Esto incluye a los sistemas de salud que se encuentran en constante cambio debido a distintos factores como las presiones económicas, una mayor exigencia y demanda por parte de los usuarios; además de la competencia, que viene a ser el sector privado del rubro de la salud ${ }^{(1)}$.

Hoy en día existe una gran competencia entre empresas, lo que lleva a ampliar ventajas competitivas. La calidad del servicio es una de las ventajas que se pueden desarrollar con completa facilidad, siempre y cuando, se sigan correctamente las políticas establecidas de atención al cliente ${ }^{(2)}$.

De la misma forma, la satisfacción del paciente representa la evaluación de la atención recibida, la cual se basa en la percepción que se tenga frente al hecho de que sus expectativas de recibir una buena atención sean cumplidas ${ }^{(3)}$.

El rubro de la medicina estética no es ajeno a estos cambios, ya que al día de hoy podemos decir que la belleza, en cierto sentido, se ha democratizado. La presión por el aspecto y el cómo se luce es grande, y las técnicas científicas han evolucionado hasta el punto de ser parte de la vida cotidiana. Lucir una piel sana y joven se ha convertido en los últimos años en uno de los motivos de consulta más frecuentes en los centros de estética, por lo que se necesitan procedimientos de calidad que contribuyan y satisfagan las expectativas de esa creciente población de clientes de las diferentes instituciones que prestan estos servicios ${ }^{(4)}$.

En los últimos años, la población peruana muestra una mayor tendencia a interesarse por el cuidado de la apariencia física personal, lo que ha convertido al Perú en un lugar de turismo estético. Sin embargo, la investigación académica realizada en nuestro país es escasa; la mayor cantidad de publicaciones procede de Colombia, si consideramos solo a los países sudamericanos de habla hispana ${ }^{(5)}$.

La institución donde se realizó este estudio es una clínica de cirugía plástica y medicina estética que se encuentra en el distrito de Jesús María (Lima, Perú). En este centro se ha observado que la calidad del servicio que brinda actualmente presenta dificultades que pueden solucionarse, por ejemplo, las demoras para la atención en la consulta, la gestión administrativa, la falta de seguimiento de pacientes, la falta de protocolos de los tratamientos, y la información incompleta que se da en consulta y que causa dudas en los pacientes, todo ello repercute negativamente en la satisfacción de los pacientes. Frente a ello se evidencia la pérdida de pacientes, las quejas y malas recomendaciones; por esta razón nace la necesidad de establecer la relación entre calidad de servicio y satisfacción en pacientes sometidos a tratamientos estéticos faciales mínimamente invasivos.

Un paciente que decide acudir a un centro estético para verse mejor físicamente y, más aún, para poner su rostro en manos de otras personas es un cliente mucho más crítico y exigente. En ese momento, sus percepciones intervienen para evaluar la calidad de atención del servicio recibido y la satisfacción. Para ello se relacionaron dos cuestionarios importantes para cada variable: el cuestionario de satisfacción del usuario de consulta externa (SUCE) y el cuestionario para calidad de servicio en base a percepciones (SERVPERF, por sus siglas en inglés), ambos con ventajas muy claras y basados en las percepciones del usuario. El SUCE tiene un número reducido de ítems, es fácilmente comprensible para los pacientes y permite obtener una medida de la satisfacción que abarca tanto la parte asistencial como la administrativa; por su parte el SERVPERF es una escala más concisa y comprensible, y sus medidas de valoración predicen mejor la calidad de servicio.

El objetivo de este estudio fue determinar la relación que existe entre la calidad de servicio y la satisfacción en pacientes sometidos a tratamientos estéticos faciales mínimamente invasivos de una clínica privada en Lima, Perú en 2019.

\section{MATERIALES Y MÉTODOS}

\section{Diseño y población de estudio}

Es un estudio de tipo observacional, descriptivo y transversal con enfoque cuantitativo y correlacional en pacientes que se sometan a tratamientos estéticos faciales mínimamente invasivos en una clínica estética durante julio, agosto y setiembre de 2019. El muestreo fue probabilístico de tipo sistemático y la muestra final colectada fueron 123 pacientes. La muestra ha sido seleccionada de acuerdo a criterios de inclusión (pacientes nuevos varones o mujeres, mayores de 18 años atendidos en la clínica que se hayan realizado uno o más de los tratamientos mínimamente invasivos en el periodo de estudio y que hayan firmado el consentimiento informado)y los de exclusión (usuario externo que acuda como acompañante, o por motivos puramente administrativos, paciente antiguo o continuador, o que no aceptaron participar en el estudio, pacientes quirúrgicos, menores de edad y los atendidos fuera del periodo de investigación).

\section{Variables y mediciones}

Las variables del estudio fueron calidad del servicio y satisfacción del paciente. Estas variables fueron medidas 
a través de la técnica de la encuesta con los cuestionarios SERVPERF y SUCE. Ambos instrumentos fueron adaptados a la población de estudio y pasaron por una prueba piloto para corroborar su validez y confiabilidad. El cuestionario SERVPERF se utilizó para medir la calidad de servicio en base a las percepciones a través de ciertas dimensiones (fiabilidad, capacidad de respuesta, seguridad, empatía y aspectos tangibles), consta de 22 ítems medidos en la escala Likert, con puntaje de 1 al 7, con fiabilidad (Alfa de Cronbach $=0,7)$ y validez de constructo mediante el análisis factorial $(\mathrm{KMO}=0,606$ y la prueba de Bartlett $=0,0)$. El segundo instrumento fue el cuestionario SUCE que se empleó para medir la satisfacción del paciente mediante dos dimensiones: atención administrativa y atención asistencial; consta de 12 ítems, medidos en escala Likert con puntaje de 1 al 10, con una fiabilidad (Alfa de Cronbach $=0,788$ ) y validez de constructo mediante el análisis factorial (KMO de 0,737 y la prueba de Bartlett $=0,0$ ).

\section{Análisis estadístico}

Se utilizó el programa estadístico SPSS 20, y el análisis descriptivo, a través de medidas de resumen como cálculo de frecuencias simples. Asimismo, se realizó la correlación de Spearman.

\section{Consideraciones éticas}

El estudio fue autorizado por el gerente general de la clínica estética. Luego de ser informado acerca de la investigación, cada paciente consintió en participar. Se resguardó la intimidad y confidencialidad de la información personal de los participantes ${ }^{(6)}$. La recolección de datos estuvo programada para el periodo julio - setiembre del 2019.

\section{RESULTADOS}

En el estudio incluimos todos los procedimientos mínimamente invasivos que la clínica ofrece (hilos tensores, microdermoabrasión con punta de diamante, toxina botulínica, bioestimulación con plasma rico en plaquetas y ácido hialurónico). La cantidad de pacientes encuestados que se sometieron a cada uno de estos procedimientos se presenta en la tabla 1.

Tabla 1. Procedimientos estéticos faciales mínimamente invasivos

\begin{tabular}{|l|l|}
\hline \multicolumn{1}{|c}{$\begin{array}{c}\text { Procedimientos } \\
\text { mínimamente } \\
\text { invasivos }\end{array}$} & $\begin{array}{c}\text { Cantidad } \\
(\mathbf{N})\end{array}$ \\
\hline Hilos tensores & 7 \\
\hline Microdermoabrasión & \\
\hline $\begin{array}{l}\text { con punta de diamante } \\
\text { Toxina botulínica }\end{array}$ & 21 \\
\hline Bioestimulación con & 16 \\
\hline plasma rico en plaquetas & 55 \\
\hline Ácido hialurónico & 24 \\
\hline Total & 123 \\
\hline
\end{tabular}

Se encuestaron 123 pacientes, de ellos, 28 (23,00\%) refiere haber recibido una atención de alta calidad, 71 (58,00\%) reportaron una atención de calidad media, y 24 (19,00\%) pacientes calificaron la atención recibida como baja calidad de servicio. Asimismo, en cada dimensión de la calidad de servicio predomina un nivel de atención medio: fiabilidad $\mathrm{N}=60(49,00 \%)$, capacidad de respuesta $\mathrm{N}=59(48,00 \%)$, seguridad $N=69$ (56,00\%), empatía $N=64$ (52,00\%) y aspectos tangibles $\mathrm{N}=70(57,00 \%)$. Además, se muestran los resultados referentes a la variable independiente: calidad de servicio en pacientes, en donde un $23,00 \%$ refiere haber recibido una atención de alta calidad, un $58,00 \%$ (71) califica con calidad media y un $19,00 \%$ (24) con baja calidad de servicio, predomina, claramente, la calidad media de atención.

Así mismo, se observa en las dimensiones de calidad de servicio, que en cada una de ellas predomina de igual manera una calidad de atención media: fiabilidad N = $60(49,00 \%)$, capacidad de respuesta $N=59(48,00 \%)$, seguridad $N=69$ (56,00\%), empatía $N=64(52,00 \%)$ y aspectos tangibles $\mathrm{N}=70$ (57,00 \%) (Tabla 2). 
Calidad de servicio y satisfacción de pacientes sometidos a tratamientos estéticos faciales mínimamente invasivos de una clínica privada. Lima-Perú. 2019

Tabla 2. Nivel de calidad de servicio global y por dimensiones en pacientes sometidos a tratamientos estéticos faciales mínimamente invasivos de una clínica privada (julio - setiembre 2019)

\begin{tabular}{|c|c|c|c|c|c|c|c|c|c|c|c|c|}
\hline \multirow{3}{*}{$\begin{array}{l}\text { Nivel de } \\
\text { calidad de } \\
\text { servicio }\end{array}$} & \multirow{2}{*}{\multicolumn{2}{|c|}{ Global }} & \multicolumn{8}{|c|}{ Dimensiones } & & \\
\hline & & & \multicolumn{2}{|c|}{ Fiabilidad } & \multicolumn{2}{|c|}{$\begin{array}{l}\text { Capacidad } \\
\text { de respuesta }\end{array}$} & \multicolumn{2}{|c|}{ Seguridad } & \multicolumn{2}{|c|}{ Empatía } & \multicolumn{2}{|c|}{$\begin{array}{l}\text { Aspectos } \\
\text { tangibles }\end{array}$} \\
\hline & $\mathbf{N}$ & $\%$ & $\mathbf{N}$ & $\%$ & $\mathbf{N}$ & $\%$ & $\mathbf{N}$ & $\%$ & $\mathbf{N}$ & $\%$ & $\mathbf{N}$ & $\%$ \\
\hline Alto & 28 & 23,00 & 36 & 29,00 & 34 & 28,00 & 25 & 20,00 & 29 & 24,00 & 39 & 32,00 \\
\hline Medio & 71 & 58,00 & 60 & 49,00 & 59 & 48,00 & 69 & 56,00 & 64 & 52,00 & 70 & 57,00 \\
\hline Bajo & 24 & 19,00 & 27 & 22,00 & 30 & 24,00 & 29 & 24,00 & 30 & 24,00 & 14 & 11,00 \\
\hline Total & 123 & 100,00 & 123 & 100,00 & 123 & 100,00 & 123 & 100,00 & 123 & 100,00 & 123 & 100,00 \\
\hline
\end{tabular}

En la tabla 3 se muestran los resultados referentes a la variable satisfacción en pacientes, en donde un $95 \%$ refiere haber salido con un grado de mediana satisfacción después de su atención y un $5 \%$ califica con satisfacción baja, predominando también una satisfacción media. Así mismo, se observa en las dimensiones de satisfacción, que en cada una de ellas predomina una satisfacción media: atención administrativa con 52 \% (64) y atención asistencial con $50 \%$ (62).

Tabla 3. Nivel de satisfacción global y por dimensiones en pacientes sometidos a tratamientos estéticos faciales mínimamente invasivos de una clínica privada (julio - setiembre 2019)

\begin{tabular}{|c|c|c|c|c|c|c|}
\hline \multirow{3}{*}{$\begin{array}{l}\text { Nivel de } \\
\text { satisfacción }\end{array}$} & \multirow{2}{*}{\multicolumn{2}{|c|}{ Global }} & \multicolumn{4}{|c|}{ Dimensiones } \\
\hline & & & \multicolumn{2}{|c|}{$\begin{array}{c}\text { Atención } \\
\text { administrativa }\end{array}$} & \multicolumn{2}{|c|}{$\begin{array}{l}\text { Atención } \\
\text { asistencial }\end{array}$} \\
\hline & $\mathrm{N}$ & $\%$ & $\mathrm{~N}$ & $\%$ & $\mathbf{N}$ & $\%$ \\
\hline Alto & 0 & 0,00 & 0 & 0,00 & 0 & 0,00 \\
\hline Medio & 117 & 95,00 & 117 & 95,00 & 117 & 95,00 \\
\hline Bajo & 6 & 5,00 & 6 & 5,00 & 6 & 5,00 \\
\hline Total & 123 & 100,00 & 123 & 100,00 & 123 & 100,00 \\
\hline
\end{tabular}

Dado que no se cumplió el requisito de normalidad (Tabla 4) para analizar la relación entre las variables se utilizó la prueba de correlación de Spearman (Tabla 5).

Tabla 4. Prueba de normalidad Kolmogorov - Smirnov

\begin{tabular}{|lcccc|}
\hline & & & & Distribución \\
& Estadistico & gl & Sig. & \\
\hline Fiabilidad & 0,168 & 123 & 0,000 & No normal \\
\hline Capacidad de respuesta & 0,214 & 123 & 0,000 & No normal \\
\hline Seguridad & 0,185 & 123 & 0,000 & No normal \\
\hline Empatía & 0,197 & 123 & 0,000 & No normal \\
\hline Aspectos tangibles & 0,160 & 123 & 0,012 & No normal \\
\hline Calidad de servicio & 0,092 & 123 & 0,000 & No normal \\
\hline Satisfacción del paciente & 0,323 & 123 & 0,000 & No normal \\
\hline
\end{tabular}




\begin{tabular}{llcccc} 
& & & & Distribución \\
& Estadístico & gl & Sig. & No normal \\
\hline Satisfacción por atención administrativa & 0,193 & 123 & 0,000 & No normal
\end{tabular}

La correlación es significativa al nivel 0,01 (bilateral)

La relación entre las variables calidad de servicio y satisfacción es Rho = 0,533; es decir, es una relación directa y moderada. Al tener sig. (nivel de significación) $=0,00$ podemos concluir que existe una relación significativa entre ambas variables (Tabla 5).

Tabla 5. Correlación de Spearman: calidad de servicio con satisfacción en pacientes sometidos a tratamientos estéticos faciales mínimamente invasivos de una clínica privada (julio - setiembre 2019)

\begin{tabular}{llcc} 
& & & \\
& & \\
& & & \\
& & \\
Rho de & Calidad & Coeficiente de correlación & 0,533 \\
\hline Spearman & de servicio & sig.(bilateral) & 0 \\
\hline
\end{tabular}

La correlación es significativa al nivel 0,01 (bilateral).

En la tabla 6 se observa la correlación entre cada una de las dimensiones de la variable calidad de servicio con la variable satisfacción del paciente: la dimensión fiabilidad y la variable satisfacción. Se observa una relación de $\mathrm{Rho}=0,437$; que significa que la relación es directa y moderada y el nivel de significación es 0,00 ; por lo que se concluye que existe una relación significativa entre ambas variables. Entre la dimensión capacidad de respuesta y la variable satisfacción existe una relación directa y moderada $(\mathrm{Rho}=0,585)$ y un nivel de significancia de 0,00 por lo que existe una relación significativa entre ambas variables. La relación entre la dimensión seguridad y la variable satisfacción es directa y casi nula (Rho $=0,097$ ) y el nivel de significación es 0,287 por lo que se concluye que existe una relación significativa entre ambas variables. Además, la relación entre la dimensión empatía y la variable satisfacción es directa y casi nula (Rho $=0,1159)$ y entre ellas no existe una relación significativa (0,078 de nivel de significación). Finalmente, entre la variable satisfacción y la dimensión aspectos tangibles se observa una relación directa y casi moderada ( $R$ ho $=0,449$ ) y un nivel de significación de 0,00 que, al ser menor de 0,05 muestra que entre ambas variables existe una relación significativa.

Tabla 6. Correlación de Spearman: dimensiones de la variable calidad de servicio con satisfacción en pacientes sometidos a tratamientos estéticos faciales mínimamente invasivos de una clínica privada (julio - setiembre 2019)

\begin{tabular}{|c|c|c|}
\hline $\begin{array}{c}\text { Dimensiones de la } \\
\text { variable calidad } \\
\text { de servicio }\end{array}$ & & $\begin{array}{c}\text { Satisfacción del } \\
\text { paciente }\end{array}$ \\
\hline \multirow[t]{4}{*}{ Fiabilidad } & Coeficiente de correlación & 0,437 \\
\hline & sig.(bilateral) & 0 \\
\hline & $N$ & 123 \\
\hline & Coeficiente de correlación & 0,585 \\
\hline \multirow[t]{3}{*}{ Capacidad de respuesta } & sig.(bilateral) & 0 \\
\hline & $\mathrm{N}$ & 123 \\
\hline & Coeficiente de correlación & 0,097 \\
\hline
\end{tabular}


Calidad de servicio y satisfacción de pacientes sometidos a tratamientos estéticos faciales mínimamente invasivos de una clínica privada. Lima-Perú. 2019

\begin{tabular}{|c|c|c|c|}
\hline & $\begin{array}{c}\text { Dimensiones de la } \\
\text { variable calidad } \\
\text { de servicio }\end{array}$ & & $\begin{array}{l}\text { Satisfacción del } \\
\text { paciente }\end{array}$ \\
\hline Rho de & Seguridad & sig. (bilateral) & 0,287 \\
\hline \multirow[t]{7}{*}{ Spearman } & & $\mathrm{N}$ & 123 \\
\hline & & Coeficiente de correlación & 0,159 \\
\hline & Empatía & sig.(bilateral) & 0,078 \\
\hline & & $\mathrm{N}$ & 123 \\
\hline & & Coeficiente de correlación & 0,449 \\
\hline & Aspectos tangibles & sig.(bilateral) & 0 \\
\hline & & $\mathrm{N}$ & 123 \\
\hline
\end{tabular}

La correlación es significativa al nivel 0,01 (bilateral)

\section{DISCUSIÓN}

Los resultados de esta investigación difieren con el trabajo de Valle et al. (7) que realizaron un estudio sobre el grado de satisfacción de los pacientes intervenidos en cirugía mayor ambulatoria en un hospital de Madrid y reportaron que el $98,4 \%$ de los pacientes se sintieron satisfechos con la atención recibida. Ayala et al. ${ }^{(8)}$ encontraron que, aproximadamente, el $70 \%$ de los pacientes presentaron un alto nivel de satisfacción; por otro lado, en la investigación de Del Salto ${ }^{(9)}$ acerca de la evaluación de la calidad de atención y satisfacción del usuario que asiste a la consulta se encontró que un $92 \%$ de los pacientes manifestaron una alta satisfacción en la atención. En 2014, Borré et al. (10), en su investigación sobre calidad percibida de la atención de enfermería, halló que el $96,8 \%$ de los pacientes se sintieron satisfechos con la atención recibida.

La investigación de Torres y León ${ }^{(11)}$ sobre el nivel de satisfacción de los pacientes atendidos en un servicio de ortodoncia encontró que el 84 \% presentó un alto nivel de satisfacción en la atención recibida y que había una relación directa entre la dimensión "elementos tangibles de la calidad de servicio" con la satisfacción del usuario; mientras que el estudio de Córdova et al. ${ }^{(12)}$ sobre satisfacción de pacientes que acuden a un centro odontológico halló que el $96,7 \%$ de participantes mostró un nivel de satisfacción de la atención calificado como bueno, y el 23,3\% como regular. Zamora ${ }^{(13)}$, en su investigación acerca de la satisfacción sobre la infraestructura y la calidad de atención en la consulta de ginecología y obstetricia, halló que el grado de satisfacción de los usuarios externos acerca de los aspectos tangibles y de la calidad de atención fue elevado. A diferencia de estos estudios, el nuestro encontró que predomina un nivel de satisfacción media, por lo que se debería mejorar la atención administrativa y asistencial, y enfocarse en las dimensiones de satisfacción empleadas.
Nuestro reporte concuerda con trabajos como el de Quispe (14) que investigó sobre calidad de servicio y satisfacción del usuario y encontró una relación significativa entre calidad de servicio y satisfacción del usuario, que demuestra una correlación positiva moderada entre las dos variables $(0,555)$. Bernedo ${ }^{(15)}$, en su estudio sobre la satisfacción por la calidad de atención y su relación con el tiempo de espera en los usuarios, encontró que un numeroso grupo de usuarios externos se sintieron satisfechos por la atención de calidad recibida en consulta, en las dimensiones de fiabilidad, capacidad de respuesta y aspectos tangibles. La investigación de Fernández ${ }^{(16)}$ encontró que la calidad de atención se relaciona con el grado de satisfacción sea esta positiva o negativa. Urure (17) halló que el nivel de satisfacción en relación a las percepciones resultó satisfecho con 4,12 de promedio en su estudio sobre satisfacción de los pacientes con la atención de enfermería postoperatoria. Asimismo, la investigación de Redhead ${ }^{(18)}$ sobre calidad del servicio y satisfacción del usuario mostró una relación significativa entre ambas variables; mientras que Vásquez y López ${ }^{(19)}$ encontraron que existe una insatisfacción moderada en todas las dimensiones de la calidad del servicio (fiabilidad, capacidad de respuesta, empatía, seguridad y aspectos tangibles) debido a deficiencias encontradas y concluyen que existe una relación significativa entre dichas variables. El estudio de Morales et al. ${ }^{(20)}$ sobre el nivel de satisfacción del paciente en un servicio de urgencias frente a la atención de enfermería reportó que el $60 \%$ expresó un nivel de satisfacción media en relación a la atención recibida, seguidos del $25 \%$ que manifiestan un nivel de satisfacción alto y el $15 \%$ restante refiere un nivel bajo.

Este estudio encontró una relación directa entre la calidad del servicio y la satisfacción del paciente; lo que explica claramente que el primero conlleva al siguiente; para ello 
debemos enfocarnos en mejorar cada dimensión de la calidad de servicio y ofrecer una alta calidad de atención y satisfacción.

Contribuciones de los autores: Todos los autores de la investigación han participado en cada etapa del estudio: la concepción, diseño del estudio, recolección de datos, obtención de resultados, análisis e interpretación de los datos, redacción del informe, revisión crítica del manuscrito, asesoría estadística, técnica o administrativa, y aprobación de versión final informe.

Fuentes de financiamiento: Este artículo ha sido financiado por los autores.

Conflictos de interés: Los autores declaran no tener ningún conflicto de interés.

\section{REFERENCIAS BIBLIOGRÁFICAS}

1. Quispe Y. Calidad de servicio y satisfacción del usuario en el servicio de traumatología del Hospital Nacional Hugo Pesce Pecetto-2015 [Tesis de pregrado]. Perú: Universidad Nacional José María Arguedas, Andahuaylas; 2015.

2. Solórzano G, Nereida J. Importancia de la calidad del servicio al cliente para el funcionamiento de las empresas. El Buzón de Pacioli. 2013; 82(5-13).

3. Córdova D, Fernández $Y$, Ortiz R. Satisfacción de los pacientes que acuden a la clínica odontológica de la Universidad de San Martín de Porres, Chiclayo, 2015. KIRU. 2016; 13(2): 132-7.

4. Torres R. Plan de mejoramiento continuo en la calidad de los procedimientos estéticos no invasivos [Tesis de postgrado]. Colombia: Universidad Santo Tomás, Bogotá; 2017.

5. Abregú J, De la Cruz D, Ying C, Gordillo V. Plan de negocio para la creación de un servicio de medicina estética en la Clínica Santa María Magdalena en la ciudad de Ayacucho - Perú [Tesis de postgrado]. Perú: Universidad ESAN, Lima; 2018.

6. Asociación Médica Mundial. Declaración de Helsinki de la AMM: Principios éticos para las investigaciones médicas en seres humanos [Internet]. 2013. Disponible en: https://www.wma.net/ es/policies-post/declaracion-de-helsinki-de-la-ammprincipioseticospara-las-investigaciones-medicas-en-sereshumanos

7. Valle M, López M, Manera I, Zazo M, Expósito A. Estudio del grado de satisfacción de los pacientes intervenidos en cirugía mayor ambulatoria en un periodo de seis meses. Rev Cir Mayor Ambulatoria. 2011; 16(4): 164-7.

8. Ayala F. Satisfacción estética, funcional y económica generada por rehabilitación con arco corto y prótesis parcial removible en pacientes de la Facultad de Odontología de la Universidad de El Salvador [Tesis de postgrado]. El Salvador: Universidad del Salvador, San Salvador; 2015.

9. Del Salto E. Evaluación de la calidad de atención y satisfacción del usuario que asiste a la consulta en el departamento médico del Instituto Nacional de Mejía en el periodo 2012 [Tesis de postgrado]. Ecuador: Universidad Central del Ecuador, Quito; 2014.

10. Borré Y, Vega Y. Calidad percibida de la atención de enfermería por pacientes hospitalizados. Ciencia y Enfermería. 2014; 20(3): 81-94.

11. Torres $G$, León $R$. Nivel de satisfacción de los pacientes atendidos en el Servicio de Ortodoncia de una Clínica Dental Docente peruana. Rev Estomatología Peru. 2015; 25(2): 122-32.
12. Córdova D, Fernández Y, Ortiz R. Satisfacción de los pacientes que acuden a la clínica odontológica de la Universidad de San Martín de Porres-Chiclayo. KIRU. 2016; 13(2): 132-7.

13. Zamora S. Satisfacción sobre la infraestructura y la calidad de atención en la consulta de Gineco-Obstetricia de un establecimiento de atención primaria. Horiz Méd. 2016; 16(1): 38-47.

14. Quispe Y. Calidad de servicio y satisfacción del usuario en el servicio de traumatología del Hospital Nacional Hugo Pesce Pecetto - 2015 [Tesis de pregrado]. Perú: Universidad Nacional José María Arguedas, Andahuaylas; 2015.

15. Bernedo R. Satisfacción por la calidad de atención y su relación con el tiempo de espera en los usuarios externos del Instituto Nacional de Oftalmología-2016 [Tesis de postgrado]. Perú: Universidad Nacional Mayor de San Marcos, Lima; 2017.

16. Fernández R. Calidad de la atención y grado de satisfacción del paciente cardiaco transferido de provincia a la consulta externa de cardiología del INCOR 2009 [Tesis de postgrado]. Perú: Universidad Nacional Mayor de San Marcos, Lima; 2014.

17. Urure I. Satisfacción de los pacientes con la atención de enfermería postoperatoria en el Servicio de Cirugía del Hospital "Santa María del Socorro" de Ica, 2006 [Tesis de postgrado]. Perú: Universidad Nacional Mayor de San Marcos, Lima; 2007.

18. Redhead R. Calidad de servicio y satisfacción del usuario en el Centro de Salud Miguel Grau Distrito de Chaclacayo 2013 [Tesis de postgrado]. Perú: Universidad Nacional Mayor de San Marcos, Lima; 2015.

19. Vásquez A, López J. Propuesta de mejora de la calidad de servicio en el policlínico Chiclayo Oeste ubicado en la ciudad de Chiclayo, durante el período 2013 [Tesis de pregrado]. Perú: Universidad Católica Santo Toribio de Mogrovejo, Chiclayo; 2015.

20. Morales $C$. Nivel de satisfacción de los pacientes que asisten al servicio de urgencias, frente a la atención de enfermería en una institución de primer nivel de atención en salud, en Mistrato Risaralda. Febrero a abril 2009 [Tesis de pregrado]. Colombia: Pontificia Universidad Javeriana, Santa Fe de Bogotá D. C.; 2009.

\section{Correspondencia:}

Karen Lissette Ramos Miranda

Dirección: Jr. Los ruiseñores Mz J1 Lote $9^{a}$ 2do piso. Urb los Jardines de San Juan. San Juan de Lurigancho. Lima, Perú. Teléfono: 934508085

Correo electrónico: karen5493@hotmail.com

Recibido: 02 de junio de 2020

Evaluado: 05 de julio de 2020 Aprobado: 24 de julio de 2020

(c) La revista. Publicado por Universidad de San Martín de Porres, Perú. (cc) Br Licencia de Creative Commons Artículo en acceso abierto bajo términos de Licencia Creative Commons Atribución 4.0 Internacional. (http://creativecommons.org/licenses/by/4.0/)

\section{ORCID iDs}

\begin{tabular}{|c|c|}
\hline Karen Lissette Ramos Miranda & (D) https: / /orcid.org/0000-0001-5231-7868 \\
\hline Luis Enrique Podesta Gavilano & (D) https: //orcid.org/0000-0003-0122 \\
\hline Raúl Alberto Ruiz Arias & (1) https://orcid.org/0000-0002-8877-6 \\
\hline
\end{tabular}

